\begin{tabular}{|c|l|}
\hline Title & $\begin{array}{l}\text { Multidirectional flexibility analysis of anterior and posterior lumbar artificial disc reconstruction: in vitro human } \\
\text { cadaveric spine model. }\end{array}$ \\
\hline Author(s) & $\begin{array}{l}\text { Kotani, Y oshihisa; Cunningham, Bryan W.; A bumi, Kuniy oshi; D mitriev, A nton E.; Hu, Niabin; Ito, Manabu; } \\
\text { Shikinami, Y Ysuo; McA fee, Paul C.; Minami, A kio }\end{array}$ \\
\hline Citation & $\begin{array}{l}\text { European Spine Journal, 15(10), 1511-1520 } \\
\text { https://doi.org/10.1007/300586-006-0086-z }\end{array}$ \\
\hline Issue Date & 2006 \\
\hline Doc URL & http://hdl.handle.net/2115/14768 \\
\hline Rights & The original publication is available at www.springerlink.com \\
\hline Type & article (author version) \\
\hline File Information & ESJ.pdf \\
\hline
\end{tabular}

Instructions for use 


\title{
MULTIDIRECTIONAL FLEXIBILITY ANALYSIS OF ANTERIOR AND POSTERIOR LUMBAR ARTIFICIAL DISC RECONSTRUCTION: IN VITRO HUMAN CADAVERIC SPINE MODEL
}

\author{
Yoshihisa Kotani, M.D.*, Bryan W. Cunningham, M.S.**, Kuniyoshi Abumi, M.D.*, \\ Anton E. Dmitriev, B.S.**, Niabin Hu, M.D.**, Manabu Ito, M.D.* \\ Yasuo Shikinami,Ph.D.***, Paul C. McAfee, M.D.**, Akio Minami, M.D.* \\ *: Dept. of Orthopaedic Surgery, Hokkaido University Graduate School of Medicine, Kita-15, \\ Nishi-7, Kitaku, Sapporo 060-8638 JAPAN, \\ **: Orthopaedic Research Laboratory, Union Memorial Hospital, 201 E. Univ. Parkway, Baltimore, \\ Maryland 21218 USA \\ ***: Takiron Co., LTD., Medical division, 2-3-13, Azuchi-machi, Chuoku, Osaka 541-0052
}

Corresponding author: Yoshihisa Kotani,M.D.

Dept. of Ortho. Surg., Hokkaido University Graduate School of Medicine

Kita-15, Nishi-7, Kitaku, Sapporo 060-8638, Japan, Tel: 81-11-706-5934, Fax:81-11-706-6054,

E-mail:y-kotani@med.hokudai.ac.jp

Acknowledgement: The study was supported by the foundation of advanced technology initiative for new industry creation of Japan and Japanese national grant in aid of scientific research No. 08407045 and 12770775.

Running Head: Multidirectional flexibility of lumbar disc replacement 


\section{Abstract}

The in vitro multidirectional flexibility analysis was conducted to investigate the initial biomechanical effect of biomimetic artificial intervertebral disc replacement from either anterior or posterior approach in a cadaveric lumbosacral spine model.

Two designs of anterior total and posterior subtotal artificial discs were developed using bioactive three-dimensional fabric and bioresorbable hydroxyapatite/poly-l-lactide (HA/PLLA) material (3DF disc). Both models were designed to obtain the stable interface bonding to vertebral endplates with maximum surface area occupation. Using seven cadaveric lumbosacral spines, the following three anterior reconstruction methods were sequentially performed at L4-5 level: anterior 3DF disc replacement; anterior BAK cages (BAK); and posterior pedicle screw fixation and anterior BAK cages combined (BAK+ PS). The L2-3 level received two methods of posterior reconstructions: subtotal 3DF disc replacement (two implants), and posterior interbody cages and pedicle screw fixation (PLIF). Six unconstrained pure moments were applied and three-dimensional segmental motions were measured with an optoelectronic motion measurement system. The center of rotation (COR) calculation was conducted radiographically using flexion-extension films. Both anterior and posterior 3DF replacements demonstrated statistically equivalent ROMs in all loading modes compared to intact segment. Anterior BAK, BAK+ PS, and PLIF demonstrated significantly lower ROMs when compared to intact and 3DF groups $(\mathrm{P}<0.05)$. The 3DF reconstruction tended to realign the COR to the posterior third or surrounding position at the operative disc level.

The stand-alone lumbar 3DF disc replacement demonstrated biomechanical 
characteristics nearly equivalent to the intact spinal segments even through anterior or posterior approach in vitro, suggesting an excellent clinical potential.

Key words: Artificial intervertebral disc, Lumbar spine, Biomechanics 


\section{Introduction}

Recent artificial intervertebral disc technology serves as the next frontier in the surgical management of intervertebral disc pathology [2,3,5,6,8,11,14-23,29,30,33,37]. As an alternative to arthrodesis, an artificial disc serves to replace the symptomatic disc, and restores the functional mobility and disc height of the motion segment. In the lumbar spine, the great attention has been focused on the adjacent segment disease after anterior and posterior spinal arthrodesis [12,27]. Etebar and Cahill reported an appropriate annual incidence of adjacent segment disease in 3.9\% and an overall prevalence of $14.4 \%$ for 125 patients followed a minimum of 3.7 years [12]. The artificial intervertebral disc may have a potential to reduce the occurrence of adjacent disc disease in a long-term period, thereby minimizing re-operations due to a new pathology.

The authors' artificial intervertebral disc is based on the concept of a durable and biomimetic design with surface modification that enables a biologic bonding to the vertebral body (Figure 1). It consists of a triaxial three-dimensional polymer fabric (3-DF) woven by an ultra-high molecular weight polyethylene (UHMWPE) fiber, and spray-coated bioactive ceramics on the disc surface $[17,20,21,29-33]$. The previous studies have demonstrated that its biocompatibility, endurance, and biomechanical property were equivalent to those of the normal disc $[17,20,21,29,30]$. The in vivo study using a sheep model demonstrated the excellent interface bonding and preservation of segmental spinal mobility up to two years 
period [20,21]. Importantly, 3DF disc is the first to possess two device designs of anterior total and posterior subtotal disc. The latter device design can achieve a posterior neural decompression and simultaneous intervertebral disc reconstruction via posterior approach for various degenerative lumbar disorders.

Although there are many lumbar artificial discs reported, only a few in vitro studies have been conducted to clarify the biomechanical properties of replaced spinal segments with recent research modalities $[9,22]$. There is still a paucity of information regarding multidirectional flexibility changes of lumbar artificial intervertebral disc replacement when compared to intact spine and other conventional spinal reconstruction procedures.

To investigate the initial biomechanical effect of lumbar artificial intervertebral disc replacement via anterior or posterior approach, the multidirectional flexibility of replaced spinal segment was analyzed using a cadaveric lumbosacral spine model. 


\section{MATERIALS AND METHODS}

\section{Design and biomechanical properties of artificial intervertebral disc (3DF disc)}

The triaxial three-dimensional fabric disc was a semi-elliptically shaped near-net woven with an ultra-high molecular weight polyethylene (UHMWPE) fiber bundle, which was coated by linear low density polyethylene [17,20,21,29,30]. The 3DF disc consisted of a number of fibers in the x-, y-, and z-axes and their respective multilayers with some alignment ratios in three dimensions. To enhance an initial fixation to vertebral endplate, two ultra-high strength bioresorbable pins made of hydroxyapatite/poly-l-lactide composite (HA/PLLA) were placed near the center of the prosthesis [31,32]. The bioactive ceramics granules were spray-coated to the designed depth with particulate unsintered hydroxyapatite (u-HA) to promote the osteointegration to the bony endplates. Several human 3DF prototypes were woven with orthogonal or off-angle fiber alignment and received cyclic tensile-compressive and torsional tests. And finally, the off-angle 45 degree model was selected based on a superior torsional property to the orthogonal and off-angle 30 degree models [17,21,29,30]. The arrangement of layer numbers and alignment ratio among three weaving axes resulted in balanced mechanical properties. The subtotal disc was designed to reconstruct the degenerative disc through posterior approach with separated two parts. This is inserted after total nucleotomy and cartilagenous endplate resection to obtain the stable interface bonding as well as initial fixation stability with bioresorbable pins. 


\section{Specimen preparation}

A total of seven fresh-frozen human cadaveric lumbosacral spines (L1-S1) were harvested en-bloc and utilized in this investigation (71 yrs old in average). The specimens were immediately packed in double-thickness plastic bags and stored -20 Celsius. Prior to biomechanical analysis, standard anteroposterior and lateral plain films were obtained to exclude specimens demonstrating malignant tumors, infections, spondylolisthesis, spondylolysis, and severe degenerative changes. In preparation for biomechanical testing, the specimens were thawed to room temperature and cleaned of all residual musculature, with care taken to preserve all ligamentous attachments and facet joint capsules. The proximal (T12-L1) and distal (Sacrum) ends of the specimen were secured in rectangular metal containers using eight compression screws and cross-fixed Steinmann pins, respectively, for fixation. The plexiglas motion detection flags were then placed on the anterior aspects of L1 to L5 vertebral bodies. Each flag was equipped with three non-co-linear light emitting diodes designed for detection by an optoelectronic motion measurement system (3020 Optotrak System, Northern Digital Inc., Waterloo, Ontario). The posterior reconstructions were performed first at L2-3 level followed by the anterior reconstructions at L4-5. Importantly, the superior container vertebral attachments were re-positioned from L1 to L3 between posterior and anterior reconstructions. 


\section{Three-dimensional flexibility testing}

Testing was performed using a custom designed six-degree-of-freedom spine simulator (6DOF-SS) configured with an Optotrak 3020 motion analysis system (Northern Digital Inc., Waterloo, Ontario). The 6DOF-SS apparatus is configured with three independent stepper motors, harmonic drives and electromagnetic clutches, which are capable of applying pure, unconstrained rotational moments $( \pm)$ about three axes - X, Y and Z. Unconstrained translations $( \pm$ ) were permitted using linear bearing guide rails (X and Z) and MTS actuator (Y axis) (Figure 2). To determine the multidirectional flexibility, non-destructive, unconstrained loading parameters included six pure moments - flexion and extension $( \pm X$ axis), lateral bending ( $\pm \mathrm{Z}$ axis) and axial rotation ( $\pm \mathrm{Y}$ axis) applied to the superior end of the vertically oriented specimen while the caudal portion of the specimen remained fixed to a testing platform. A maximum applied moment of $\pm 8 \mathrm{Nm}$ was used for each loading mode and applied at a stepper motor rate of three degrees/second. A total of three load / unload cycles was performed for each motion with data analysis based on the final cycle. For the six main motions - corresponding to the moments applied - the operative level vertebral rotations (degrees) were quantified in terms of peak range of motion (ROM) and neutral zone (NZ). ROM is defined as the peak displacement from the initial neutral position to maximum load, while NZ represents the motion from the initial neutral position to the unloaded position at the beginning of the third cycle [25]. 


\section{Surgical reconstruction groups}

Following analysis of the intact spine, each specimen was reconstructed at the L2-3 and L4-5 intervertebral levels according to the following group assignments:

\section{Posterior reconstruction group at L2-3 (Figure 3)}

1) Bilateral partial medial facetectomies and diskectomy (no testing)

2) Subtotal 3DF posterior disc replacement (two implants)

3) Posterior interbody fusion cages (Brantigan cage) and VSP pedicle screw fixation (Depuy-AcroMed, Inc.).

For implantation of 3DF device, bilateral partial medial facetectomies and the resection of supraspinous and interspinous ligaments were performed at the L2-3 level, followed by the diskectomy. Two 3DF posterior implants (13 x 22 x H10mm) were then implanted with special distracter and inserter, after applying the adequate distraction force and assuring the anatomical device fit (Figure 3A). After testing, the 3DF implants were carefully removed and replaced with two appropriately sized Brantigan cages. VSP pedicle screws and plates were then implanted to produce a 360 degree reconstruction and the construct re-tested (Figure 3B).

\section{Anterior reconstruction group at L4-5 (Figure 4)}

1) Anterior diskectomy (no testing)

2) Anterior total 3DF disc replacement (single implant)

3) Anterior BAK device (two anterior cages) (Centerpulse SpineTech, Inc.)

4) Anterior BAK cages and posterior VSP pedicle screw fixation (360 degree).

Following the completion of the posterior implant testing, the specimen was re-potted to 
permit biomechanical analysis of the L4-L5 operative level. After intact analysis, a complete anterior diskectomy was performed at L4-L5 followed by curettage of the vertebral endplates to permit implantation of anterior 3DF device. The diskectomy procedure included removal of the anterior longitudinal ligament, anterior annulus, and nucleus pulpous. For appropriate device fit with the tension of anterior longitudinal ligament and outer annulus, the combined distracter and inserter was used to place the anterior 3DF (35 x 27 x H12 or 14) (Figure 4A). For BAK Device testing, the anterior 3DF implant was carefully removed and replaced with two appropriately sized BAK implants at L4-L5 (Figure 4B). For the final test, VSP pedicle screws and posterior plates were implanted at L4-L5 to produce a 360 degree reconstruction and the construct re-tested.

\section{Radiographic analyses on Center of Rotation (COR) calculation}

To determine the centers of intervertebral rotation at the lumbar operative level, three stepwise lateral plain films (full flexion, neutral and full extension) were obtained for each spinal reconstruction condition. These films were subsequently digitized with two combinations of flexion-extension and neutral-extension, and the centers of intervertebral rotation (COR) were calculated by superimposing radiographic images of the stationary underlying vertebra at each level, and comparing the positions of identical points on the two images of the moving vertebral endplate. The procedures were repeated three times, and the average centers of rotation were represented by the point of tangency between these 
bisectors.

\section{Data and statistical analysis}

The intervertebral range of motion (ROM) at the operative L2-3 and L4-5 level was calculated as the sum of the neutral and elastic zones $(\mathrm{NZ}+\mathrm{EZ}=\mathrm{ROM})$ and represented the peak total range of motion (Euler angles rotation) at the third loading cycle. The expressed degrees of rotation (axial rotation \pm Y-axis; flexion-extension $\pm \mathrm{X}$-axis and lateral bending \pm Z-axis) for multi-directional flexibility analyses were according to the three-dimensional conceptual framework of Panjabi. ${ }^{24}$ The non-destructive range of motion data was normalized to the intact spine condition for each loading mode. Neutral zone was also expressed by normalized percent to the intact spine value. Statistical analysis included descriptives and a One-way Analysis of Variance with Student-Newman-Keuls test for group-to-group comparisons. Comparisons with values of $\mathrm{p}<0.05$ were considered statistically significant. 


\section{RESULTS}

Following all biomechanical testing, 3-DF device did not show any device loosening or dislodgement. The initial stability afforded by an effective disc height and two bioresorbable pins maintained after several cycles of testing.

\section{Operative level ROM analysis}

Reconstruction at L2-L3 with the posterior 3DF device demonstrated a statistically equivalent axial rotational motion of $117.9 \pm 23 \%$ compared to the intact condition $(\mathrm{p}>0.05)$, while the addition of Brantigan cages and pedicle screw instrumentation significantly decreased motion to $60.8 \pm 31.3 \%(\mathrm{p}<0.05)$ (Figure 5). In similarity to the results in axial rotation, the reconstruction at L2-L3 with posterior 3DF device demonstrated a statistically equivalent flexion-extension motion of $132.9 \pm 36.4 \%$ compared to the intact condition ( $>0.05$ ), while the addition of Brantigan cages augmented with pedicle screw instrumentation significantly decreased motion to $23.12 \pm 19.18 \%(\mathrm{p}<0.05)$ (Figure 5). Lateral bending of the posterior 3DF device showed an equivalent motion to $102.3 \pm 15.21 \%$ compared to the intact condition ( $\mathrm{p}>0.05)$, while the addition of Brantigan Cages augmented with pedicle screw instrumentation significantly decreased motion to $30.3 \pm 18.7 \%(\mathrm{p}<0.05)$ (Figure 5).

The anterior 3DF device increased the axial rotational motion to $146.4 \pm 58.36$ at 
L4-L5, however, this was not significantly different from the intact condition ( $p>0.05)$. The BAK cages reduced motion to $74.35 \pm 47.2 \%$, which was significantly less than the 3DF treatment $(p<0.05)$. Pedicle screw instrumentation reduced motion to $22.57 \pm 16 \%$ of the intact condition and was significantly less than all other treatments $(\mathrm{p}<0.05)$ (Figure 6). The anterior 3DF device demonstrated a statistically equivalent flexion-extension motion of $104.32 \pm 20.83 \%$, while the BAK cages reduced motion to $43 \pm 28.5 \%$ and pedicle screws with rods further reduced motion to $9.5 \pm 6.88 \%(\mathrm{P}<0.05)$. The latter two reconstructions were significantly less than the intact and 3DF reconstructions $(\mathrm{p}<0.05)$ and the pedicle screw construct was less than the BAK group $(\mathrm{p}<0.05)$ (Figure 6). The anterior 3DF device demonstrated a statistically equivalent lateral bending motion of $113.32 \pm 41.3 \%$, while the BAK cages reduced motion to $52 \pm 24.8 \%$ and pedicle screws with rods further reduced motion to $16.7 \pm 14.8 \%$. The latter two reconstructions were significantly less than the intact and 3DF reconstructions $(\mathrm{p}<0.05)$ and the pedicle screw construct was less than the BAK group $(\mathrm{p}<0.05)$ (Figure 6).

\section{Operative level NZ analysis}

The neutral zone increased for posterior 3DF disc under axial rotatation when compared to the intact value $(\mathrm{P}<0.05)$ (Figure 7). In both flexion-extension and lateral bending, the intact, 3DF, and PLIF groups formed a statistically equal subset at L2-3. Anterior 3DF 
reconstruction demonstrated 36\% of increase in axial rotation, however, there was no statistical difference among intact and three treatment groups in all loading modes. (Figure 8) In flexion-extension and lateral bending, despite the large standard deviations, the BAK and BAK combined with pedicle screw constructs significantly reduced the neutral zone region compared to the intact condition $(\mathrm{p}<0.05)$.

\section{Center of Rotation (COR) analysis}

The calculated intervertebral centers of rotation (COR) at the operative level were demonstrated for the intact spine condition and anterior 3DF reconstruction in each specimen (Figure 9). The COR locations at L4-5 levels showed wide variations and were mostly deviated from a physiological COR position of posterior third at the intervertebral disc, bordering or slightly below the vertebral endplate[6]. After the anterior 3DF replacement, the COR locations showed the tendency of realignment to the posterior third or surrounding position at operative disc levels. 


\section{Discussion}

Recent artificial disc prostheses are classified into a metal on polymer

$[4,11,13,14,23,37]$, metal on metal $[7,15,19]$, and exclusive polymer design

$[18,20,21,29,30,35,36]$. The device configuration is mostly a total replacement design from

anterior transabdominal or retroperitoneal approach $[6,8,11,14,23,37]$. The representative

metal on polymer artificial discs include SB Charite and ProDisc, in which polyethylene core

is placed between two metal endplates with unconstrained or semi-constrained design

$[6,11,14]$. McAfee et al. reported the early experience of SB Charite in 60 patients of

prospective randomized study in the United States [23]. Although overall comparison to

spinal fusion with BAK cages was not demonstrated, the initial clinical outcome was

acceptable in terms of low back pain reduction and radiographic measures. However, among

many clinical cases in Europe, some complications were reported. Van Ooij et al. reported a

series of twenty-seven patients with SB Charite disc, presenting the subsidence of the

prosthesis, anterior subluxation of polyethylene core, polyethylene wear and compression of

great vessels requiring revision surgeries [34]. This type of modular unconstrained disc

design has a possibility to cause the failure between components as well as wear debris

production. The in vivo goat study by Anderson et al. demonstrated the polarizable foreign

material and partially polarizable intracellular granular material exterior to the dura mater

and in macrophages at periprosthetic tissues with Bryan cervical disc replacement [1]. The 
significance of this wear debris cannot be determined unless cytokines are specially looked for, however, the metal on polyethylene type prosthesis has a tendency to cause wear complications as shown in general joint arthroplasty [34]. The metal on metal prosthesis was reported historically by Kostuik, and was recently introduced with Marverick disc [19,22]. In hip joints, the metal on metal joint mechanism was reported to cause an early loosening of the device in spite of many controversies, and modified to polyethylene back mechanism allowing shock absorption and stress relaxation $[10,28]$. The prospective investigational device exemption (IDE) study is under way in Marverick disc, the early clinical results should be carefully discussed in the future.

Several polymer type artificial discs were reported previously by Vuono-Hawkins and Bao, et al $[2,35,36]$. To adapt the anisotropy and high torsional stiffness of the natural intervertebral disc, the polymer composites with different materials or reinforced fibers were investigated. However, many investigators failed to gain the sufficient biomechanical properties as well as stable interface with direct bone contact between prosthesis and vertebral body [36].

The 3DF disc is the first to biomimetically control the mechanical and viscoelastic properties using a fabric woven by a monofilament involving multi UHMWPE fibers, allowing the arrangement of textile density and fiber alignment $[20,21,29,30]$. The surface bioactivity was supplemented with particulate unsintered hydroxyapatite to achieve a bone 
ingrowth to the inside of the fabric. The construction with soft organic materials will prevent surrounding soft tissue damages during insertion as well as a relative easiness of revision surgery. Another important advantage of 3DF disc is to create different device configuration with either total or subtotal device design. The artificial disc for total disc replacement anteriorly has several benefits: wide surface area occupation, excellent mechanical endurance, and wide range of motion. Surgical indications, however, are extremely limitedthat is, to application in cases of degenerative disc disease and postdiscectomy disorders without neurologic deficits and facet arthrosis. The posterior subtotal 3DF disc evaluated in this study was intended to reconstruct the degenerative intervertebral disc posteriorly with simultaneous neural decompression for further wider range of lumbar degenerative disorders. In contrast to preexisting artificial nucleus devices, this was intended to act as a load-sharing device with stable interface bonding. The surgical indications include a recurrent disc herniation and lumbar spinal canal stenosis with a slight segmental instability, and low-grade lumbar spondylolisthesis. This has a possibility to expand the pool of candidates for artificial disc replacement, however, the safety and long-term effectiveness of this technique are still unknown.

The present study investigated the initial multidirectional flexibility of artificial intervertebral disc replacement using both anterior total and posterior subtotal stand-alone models. According to other bench-top reports, anterior annular resections in total disc 
replacement increased axial rotational flexibility [9]. Cunningham et al. demonstrated 44\% increase of axial rotational flexibility in SB Charite prosthesis, which was statistically different from intact value [9]. We found a same trend of $46 \%$ increase in axial rotational ROM, however, this did not reach a statistical significance. The posterior annular resections were reported to create flexion-extension instability, and anterior column reconstructions through posterior approach tended to be inferior to intact segment without spinal instrumentations [26]. However, in this study, the average ROM values of posterior 3DF disc reconstruction demonstrated 30\% higher values to intact spine in flexion-extension loading without a statistical significance. Even repetitive biomechanical loadings did not lead to any device loosening or dislodgement.

The COR analysis after artificial disc replacement has been scarcely conducted. The present results demonstrated that the anterior 3DF replacement showed the tendency to re-align the COR locations to the posterior third or surrounding position at operative disc level. Although spines were mostly aged and degenerated, these COR trends were found in all specimens.

There are still unsolved problems in the endurance of the device at adjacent disc pathology to the multiple-level arthrodesis, and revision strategies. The use at the adjacent segment pathology to multiple segment arthrodesis will dramatically increase the mechanical stress to the artificial disc; therefore require the sufficient durability with physical dynamic 
mobility. The 3-DF disc did not cause the wear debris after 63 million alternating stresses for anti-fatigue testing, which were equivalent to natural biological movements for a period of more than 30 years [30]. The revision surgery of metal or metal on polyethylene type prosthesis requires the huge amount of bone resection as well as the technical difficulty and neurologic risk. The present prosthesis facilitates the revision surgery with simple excavation of the material. Finally, the lumbar artificial disc replacement surgery with 3DF disc has a potential to change the treatment strategy of several lumbar spine disorders using both anterior and posterior approaches. This may surpass the spinal fusion as the spinal reconstruction method of choice over existing other total artificial intervertebral discs.

\section{Conclusions}

To investigate the initial biomechanical effect of artificial intervertebral disc replacements with anterior total and posterior subtotal designs, the multidirectional flexibility of replaced spinal segment was analyzed using a cadaveric lumbosacral spine model. The stand-alone lumbar 3DF disc replacement demonstrated nearly physiological biomechanical characteristics even through anterior or posterior approach, suggesting an excellent clinical potential. 


\section{References}

1. Anderson PA, Rouleau JP, Bryan VE, Carlson CS. Wear analysis of the Bryan cervical disc prosthesis.evaluation. Spine. 2003 Oct 15;28(20):S186-94.

2. Bao QB, McCullen GM, Higham PA, Dumbleton JH, Yuan HA. The artificial disc: theory, design and materials. Biomaterials. 1996 Jun;17(12):1157-67.

3. Bao QB, Yuan HA. Prosthetic disc replacement: The future? Clin Orthop. 2002 Jan;(394):139-45.

4. Bryan VE. Cervical motion segment replacement. Eur Spine J. 2002 Oct;11 Suppl 2:S92-7.

5. Buttner-Janz K, Schellnack K, Zippel H: Biomechanics of the SB Charite lumbar intervertebral disc endoprosthesis. Int Orthop. 1989;13(3):173-6.

6. Cinotti G, David T, Postacchini F. Results of disc prosthesis after a minimum follow-up period of 2 years. Spine. 1996 Apr 15;21(8):995-1000.

7. Cummins BH, Robertson JT, Gill SS. Surgical experience with an implanted artificial cervical joint. J Neurosurg. 1998 Jun;88(6):943-8.

8. Cunningham BW, Lowery GL, Serhan HA. Total disc replacement arthoroplasty using the AcroFlex lumbar disc: a non-human primate model. Eur Spine J. 2002 Oct;11 Suppl 
2:S115-23.

9. Cunningham BW, Gordon JD, Dmitriev AE, Hu N, McAfee PC. Biomechanical evaluation of total disc replacement arthroplasty: an in vitro human cadaveric model. Spine. 2003 Oct;28:s110-117.

10. Dorr LD, Hilton KR, Wan Z. Modern metal on metal articulation for total hip replacements. Clin Orthop. 1996 Dec;(333):108-17.

11. Enker P, Steffee A, Mcmillin C, Keppler L, Biscup R, Miller S. Artificial disc replacement. Preliminary report with a 3-year minimum follow-up. Spine. 1993 Jun 15;18(8):1061-70.

12. Etebar S, Cahill DW. Risk factors for adjacent-segment failure following lumbar fixation with rigid instrumentation for degenerative instability. J Neurosurg 1999 April;90:163-169.

13. Goffin J, Casey A, Kehr P, et al: Preliminary clinical experience with the Bryan cervical disc prosthesis. Neurosurgery. 2002 Sep;51(3):840-5; discussion 845-7.

14. Griffith SL, Shelokov AP, Buttner-Janz K, LeMaire JP, Zeegers WS. A multicenter retrospective study of the clinical results of the Link SB Charite intervertebral prosthesis. Spine. 1994 Aug 15;19(16):1842-9.

15. Hedman TP, Kostuik JP, Fernie GR. Design of an intervertebral disc prosthesis. Spine. 
1991 Jun;16(6 Suppl):S256-60.

16. Hochschuler SH, Ohnmeiss DD, Guyer RD, Blumenthal SL. Artificial disc: preliminary results of a prospective study in the United States. Eur Spine J. 2002 Oct;11 Suppl 2:S106-10.

17. Kadoya K, Kotani Y, Abumi K, Takada T, Shimamoto N, Shikinami Y, Kadosawa T, Kaneda K. Biomechanical and morphologic evaluation of a three-dimensional fabric sheep artificial intervertebral disc: In vitro and in vivo analysis. Spine. 2001 Jul 15;26(14):1562-9.

18. Klara PM, Ray CD. Artificial nucleus replacement. Clinical experience. Spine. 2002 Jun 15;27(12):1374-7.

19. Kostuik JP: Intervertebral disc replacement. Experimental study. Clin Orthop. 1997 Apr;(337):27-41.

20. Kotani Y, Abumi K, Shikinami Y, Takahata M, Kadoya K, Kadosawa T, Minami A, Kaneda K. Two-year observation of artificial intervertebral disc replacement: results after supplemental ultra-high strength bioresorbable spinal stabilization. J Neurosurg. 2004 Apr;100(4 Suppl):337-42.

21. Kotani Y, Abumi K, Shikinami Y, Takada T, Kadoya K, Shimamoto N, Ito M, Kadosawa T, Fujinaga T, Kaneda K. Artificial intervertebral disc replacement using bioactive three-dimensional fabric: design, development, and preliminary animal study. 
Spine. 2002 May 1;27(9):929-35; discussion 935-6.

22. LeHuec JC, Kiaer T, Friesem T, Mathews H, Liu M, Eisermann L. Shock absorption in lumbar disc prosthesis: preliminary mechanical study. J Spinal Disord Tech. 2003 Aug;16(4):346-51.

23. McAfee PC, Fedder IL, Saiedy S, Shucosky EM, Cunningham BW. Experimental design of total disk replacement- Experience with a prospective randomized study of the SB Charite. Spine. 2003 Oct;28(20s):s153-162.

24. Panjabi MM. Biomechanical evaluation of spinal fixation devices: I. a conceptual framework. Spine. 1988 Oct;13(10):1129-34.

25. Panjabi MM. The stabilizing system of the spine. Part II. Neutral zone and instability hypothesis. J Spinal Disord. 1992 Dec;5(4):390-6; discussion 397.

26. Panjabi MM. Physical properties and functional biomechanics of the spine, in White AA III, and Panjabi MM(ed): Clinical biomechanics of the spine, ed 2. Philadelphia: Lippincott-Raven, 1990, pp 1-84.

27. Robbins MM, Hilibrand AS. Post-arthrodesis adjacent segment degeneration, in Vaccaro A, Anderson DG, Crawford A (ed): Complications of pediatric and adult spinal surgery. Philadelphia, Lippincott William \& Wlkins, 2003.

28. Schmidt M, Weber H, Schon R, et al. Cobalt chromium molybdenum metal combination 
for modular hip prosthesis. Clin Orthop. 1996 Aug;(329 Suppl):S35-47.

29. Shikinami Y, Kawarada H. Potential application of a triaxial three-dimensional fabric (3-DF) as an implant. Biomaterials. 1998 Apr-May;19(7-9):617-35.

30. Shikinami Y, Kotani Y, Cunningham BW, et al: A biomimetic artificial disc with improved properties compared to biological intervertebral discs. Adv Funct Mater 2004 (in press).

31. Shikinami Y, Okuno M: Bioresorbable devices made of forged composites of hydroxyapatite (HA) particles and poly-l-lactide (PLLA): Part I: Basic characteristics. Biomaterials. 1999 May;20(9):859-77.

32. Shikinami Y, Okuno M. Bioresorbable devices made of forged composites of hydroxyapatite (HA) particles and poly-l-lactide (PLLA): Part II: practical properties of miniscrews and miniplates. Biomaterials. 2001 Dec;22(23):3197-211.

33. Takahata M, Kotani Y, Abumi K, Shikinami Y, Kadosawa T, Kaneda K, Minami A. Bone ingrowth fixation of artificial intervertebral disc consisting of bioceramic-coated three-dimensional fabric. Spine. 2003 Apr 1;28(7):637-44; discussion 644.

34. Van Ooij A, Oner FC, Verbout AJ: Complications of artificial disc replacement. A report of 27 patients with the SB Charite disc. J Spinal Disord Tech. 2003 Aug;16(4):369-83. 
35. Vuono-Hawkins M, Langrana NA, Parsons JR, Lee CK, Zimmerman MC. Materials and design concepts for an intervertebral disc spacer II. Multidurometer composite design. J Appl Biomater. 1995 Summer;6(2):117-23.

36. Vuono-Hawkins M, Zimmerman MC, Lee CK, Carter FM, Parsons JR, Langrana NA. Mechanical evaluation of a canine intervertebral disc spacer: in vitro and in vivo studies. J Orthop Res. 1994 Jan;12(1):119-27.

37. Zeegers WS, Bohnen LM, Laaper M, Verhaegen MJ. Artificial disc replacement with the modular type SB Charite III: 2-year results in 50 prospectively studied patients. Eur Spine J. 1999;8(3):210-7. 
Figure Legend

Figure 1: Biomimetic three-dimensional fabric lumbar artificial disc (3DF disc $2^{\text {nd }}$ model). Unsintered hydroxyapatite granules were spray-coated on the surface and bioresorbable pins made of hydroxyapatite/poly-l-lactide composite (HA/PLLA) were placed near the center of the prosthesis for initial fixation (shown with arrows).

1A: Total replacement model for anterior approach

1B: Subtotal (divided) replacement model for posterior approach.

Figure 2: Oblique view of six-degree-of-freedom spine simulator (6DOF-SS) attached to the specimen. The gimbal is configured with three independent stepper motors, harmonic drives and electromagnetic clutches, which are capable of applying pure, unconstrained rotational moments about three axes- X, Y, and Z. Unconstrained translations are permitted using linear bearing guide rails (X and Z) and MTS actuator (Y axis).

Figure 3 (3A, 3B): Posterior reconstructions at L2-3 level 3A: Subtotal 3DF posterior disc replacement (two implants). Following bilateral partial medial facetectomies, the diskectomy and curettage of vertebral endplates were performed through two annular windows. Using a specially designed distracter and inserter, each 
implant strongly held the endplate with two bioresorbable pins, providing an excellent interface stability.

3B: Posterior interbody fusion cages (Brantigan cage) and VSP pedicle screw fixation.

Figure 4: Anterior reconstructions at L4-5 level.

4A: Anterior diskectomy followed by curettage of the vertebral endplates.

4B: Anterior 3DF disc insertion with a specially designed distracter and inserter. This instrument protects bioresorbable pins during insertion, and subsequently holds endplates after the release of segmental distraction.

4C: Anterior 3DF disc in place. The device perfectly fits with a large surface area occupation.

4D: Anterior BAK cage reconstruction.

Figure 5: Operative level ROMs of posterior lumbar reconstructions under axial rotation, flexion-extension, and lateral bending. Intact spine: intact L2-3 spinal segment; 3DF device: posterior 3DF disc (two implants); Pedicle screws and cages: posterior VSP pedicle screw fixation and two Branigan cages (PLIF construct). * -indicates a statistically significant difference from other two reconstruction groups with a p-value below 0.05 . 
Figure 6: Operative level ROMs of anterior lumbar reconstructions under axial rotation, flexion-extension, and lateral bending. Intact spine: intact L4-5 spinal segment; 3DF device: anterior 3DF disc reconstruction; BAK: anterior BAK cage reconstruction (two cages); Pedicle screws and cages: posterior VSP pedicle screw fixation with anterior BAK cages (360 degree reconstruction). * -significantly different from all other groups with a p-value below . ** -significantly different from 3DF group with a p-value below 0.05. \#-significantly different from 3DF and pedicle screws and cages groups with a p-value below 0.05.

Figure 7: Operative level neutral zone (NZ) of posterior lumbar reconstruction under axial rotation, flexion-extension, and lateral bending. Intact: intact L2-3 spinal segment; 3DF: posterior 3DF disc reconstruction; Pedicle screws and cages: posterior VSP pedicle screw fixation and two Branigan cages (PLIF construct). * -indicates a statistically significant difference from intact spine with a p-value below 0.05 .

Figure 8: Operative level neutral zones (NZ) of anterior lumbar reconstructions under axial rotation, flexion-extension, and lateral bending. Intact spine: intact L4-5 spinal segment; Ant 3DF: anterior 3DF disc reconstruction; BAK: anterior BAK cage reconstruction (two cages); Pedicle screw and BAK: posterior VSP pedicle screw fixation with anterior BAK cages (360 degree reconstruction). ${ }^{*}$-indicates a statistically significant difference from intact spine 
with a p-value below 0.05 .

Figure 9: Diagrammatic representation of the calculated centers of intervertebral rotation at the operative (L4-5) levels. The red and green circles signify the preoperative and postoperative COR location, respectively.

9A: Specimen \#2; 9B: Specimen \#5 


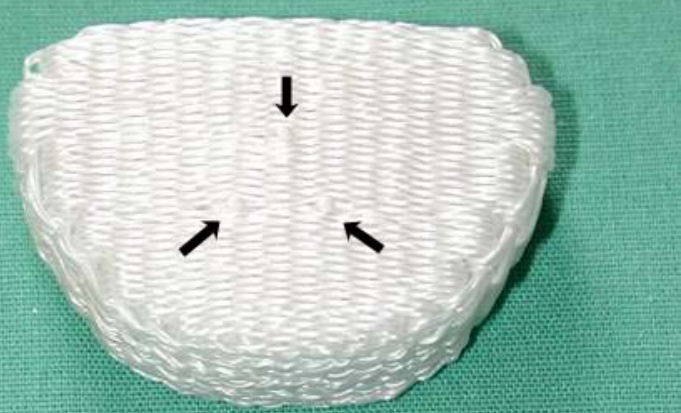

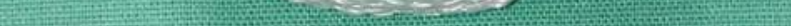

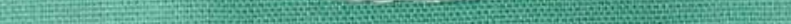
LE

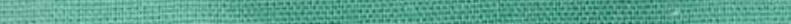
thit

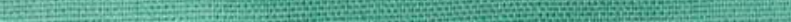

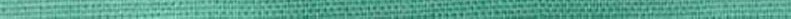






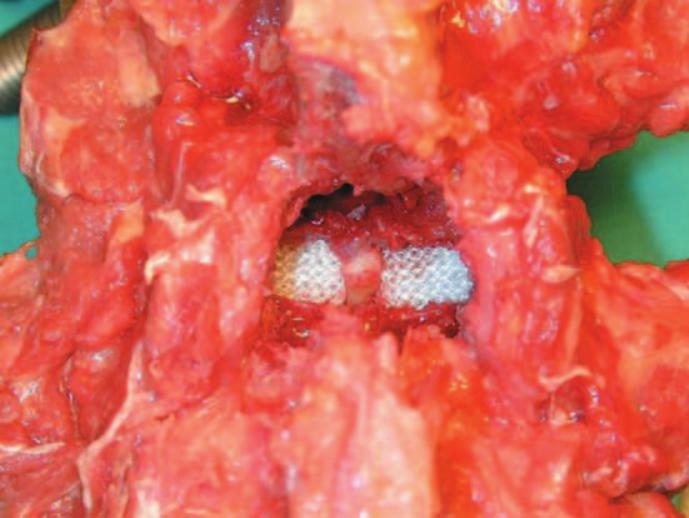





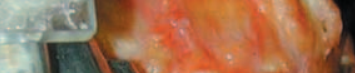




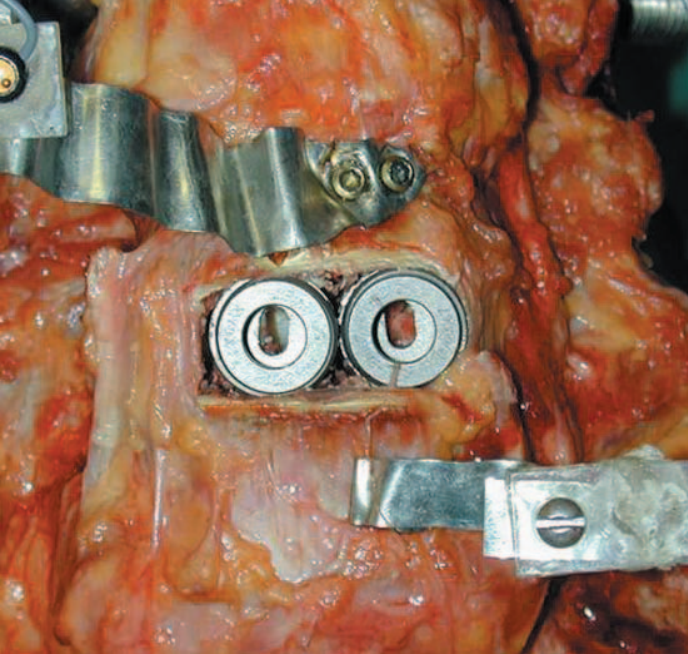




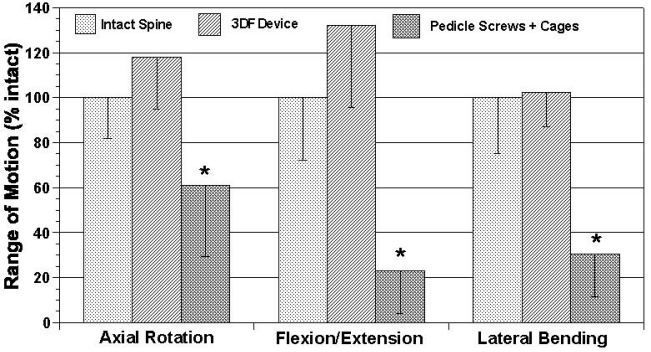

\section{Biomechanical Loading Mode}




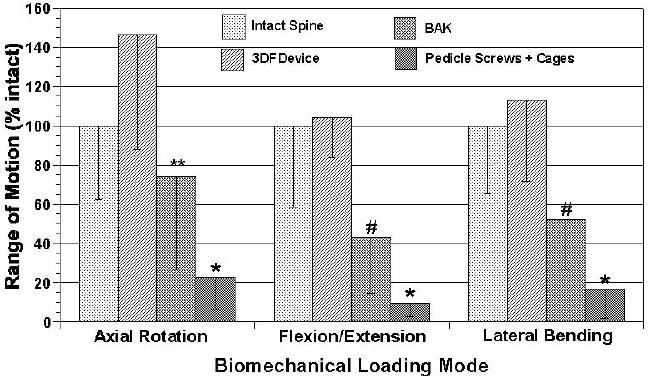




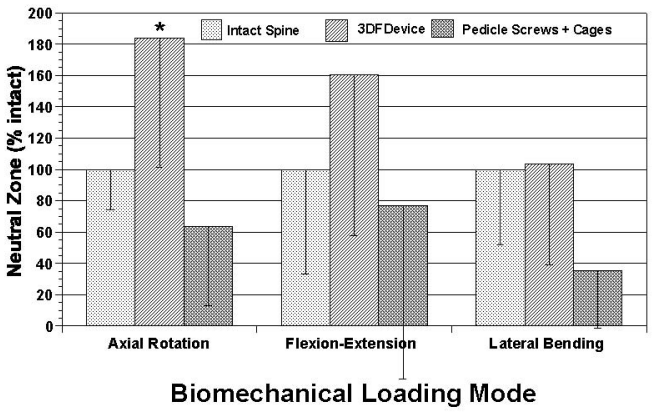




\section{Specimen \#2: 66}

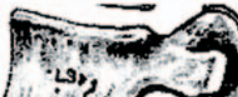

ibigitistis

inim,

[1]: is

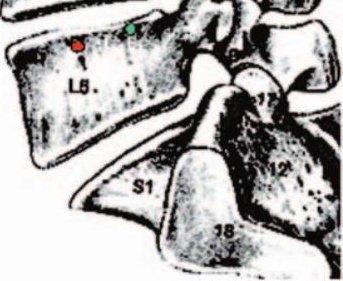




\section{Specimen \#5: 73}
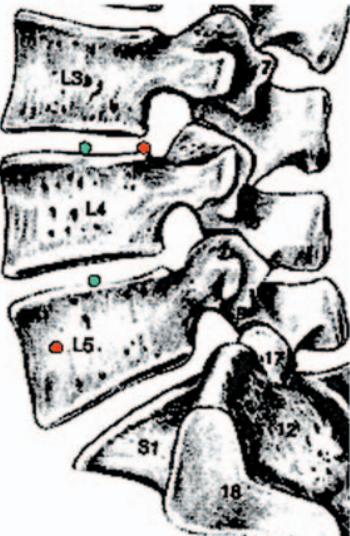
Table 1. Range of motion (ROM) and Neutral zone (NZ) of anterior and posterior reconstruction r under three biomechanical loading modes.

Biomechanical loading mode

\begin{tabular}{cccc} 
& Axial Rotation & Flexion-Extension & Lateral Bending \\
\hline ROM (deg) & & & \\
\hline L2/3 leve/ & & & \\
Intact & $7.4 \pm 1.3$ & $6.0 \pm 1.7$ & $9.2 \pm 2.3$ \\
Post 3DF & $8.6 \pm 2.1$ & $8.2 \pm 1.4$ & $10 \pm 2.3$ \\
PS + post cages & $4.4 \pm 2.6$ & $1.2 \pm 1.0$ & $2.7 \pm 1.7$ \\
\hline L4/5 level & & & \\
Intact & $3.2 \pm 1.2$ & $7.7 \pm 3.2$ & $6.9 \pm 2.4$ \\
Ant 3DF & $4.1 \pm 2.2$ & $8.7 \pm 4.3$ & $7.8 \pm 3.8$ \\
Ant BAK & $2.6 \pm 2.5$ & $3.4 \pm 3.4$ & $3.9 \pm 2.4$ \\
PS + BAK & $0.7 \pm 0.7$ & $0.7 \pm 0.6$ & $1.3 \pm 1.4$ \\
& & & \\
\hline NZ (deg) & & & \\
\hline L2/3 level & & & $2.2 \pm 1.1$ \\
Intact & $1.0 \pm 0.3$ & $1.4 \pm 0.9$ & $2.1 \pm 1.2$ \\
Post 3DF & $1.8 \pm 0.8$ & $1.8 \pm 0.9$ & $0.6 \pm 0.6$ \\
PS + post cages & $0.7 \pm 0.5$ & $0.9 \pm 1.2$ & $1.2 \pm 0.6$ \\
\hline L4/5 level & & & $1.3 \pm 0.8$ \\
Intact & $0.5 \pm 0.6$ & $2.1 \pm 2.1$ & $0.3 \pm 0.5$ \\
Ant 3DF & $0.9 \pm 1.0$ & $1.5 \pm 1.3$ & $0.1 \pm 0.2$ \\
Ant BAK & $0.3 \pm 0.3$ & $0.3 \pm 0.3$ & $($ Mean $\pm S T D)$ \\
PS + BAK & $0.1 \pm 0.2$ & $0.1 \pm 0.1$ &
\end{tabular}

